Revista de Psicología de la PUCP. Vol. XIX, 1, 2001

\title{
Soporte social en personas que viven con VIH
}

\author{
Ninoshka Fasce Cayo ${ }^{1}$
}

Se investiga el soporte social en un grupo de adultos con VIH: catorce mujeres y cuarenta y un hombres, de entre 18 y 58 años, de estrato socioeconómico bajo y medio bajo que acuden a centros de salud estatales, organismos no gubernamentales y grupos de ayuda mutua (GAM) de Lima. Se correlacionaron los resultados descriptivos de los índices soporte social percibido, soporte social efectivo, satisfacción con el soporte y necesidad de soporte con las variables edad, sexo, tiempo de diagnóstico, presencia o no de síntomas asociados al $\mathrm{VIH}$, participación o no en un GAM y orientación sexual (hombres heterosexuales, hombres que tienen sexo con otros hombres (HSH) y mujeres heterosexuales). Se encontró que los índices del soporte social se comportan diferente según se pertenezca a un GAM o no, según el sexo, según la orientación sexual y el sexo. Asimismo, el índice necesidad de soporte varía según el tiempo de diagnóstico.

Palabras clave: Soporte social, Virus de Inmunodeficiencia Humano (VIH), grupo de ayuda mutua (GAM), hombre que tiene sexo con otros hombres (HSH).

\section{Social support in people, that live with HIV in Lima}

This research studies the social support in a group of adult persons with HIV: 14 women and 41 men between 18 and 58 years of age, from medium - low social economic status that appeal to state health centers, non government entities and mutual support groups of Lima. The results of perceived social support, effective social support, social support satisfaction and social support need have been correlated with variables age, sex, diagnostic time, presence of symptoms associated to HIV, participation in a mutual support group, sex and sexual orientation (heterosexual males, men that have sex with other men and heterosexual women).

The results showed that the social support indexes behaved differently according to weather they participated in a mutual support group or not, according to sex, according to sex and sexual orientation. Also, the need for social support rate varies according to diagnostic time.

Keywords: Social support, HIV, mutual support group, man that have sex with men.

1. Licenciada en Psicología clínica de la PUCP. Es psicóloga del Grupo de Ayuda Mutua (GAM) Vivir y Presidenta de la Asociación UNESCO Warmicuna Perú. Investiga los factores que afectan la calidad de vida de las personas que viven con VIH. Correo electrónico: ninoshka@terra.com.pe. 

El VIH cuenta actualmente con 1'987,217 casos en el ámbito mundial, 951,755 en Latinoamérica (Organización Mundial de la Salud, 1998), 16,471 en el Perú, (Ministerio de Salud, 1999) y 11,709 en Lima. Sin embargo, lo que se debe considerar es la calidad de vida de los seropositivos que se ve afectada directamente por los efectos del virus sobre el sistema inmune, así como, por los sentimientos de culpa, vergüenza, cólera, ansiedad, depresión y miedo frente a la muerte. Se sabe que las emociones negativas afectan directa y negativamente la respuesta inmunológica, lo que en el caso de los seropositivos es particularmente perjudicial. En este contexto el soporte social ejerce un papel relevante al ayudar a manejar las emociones negativas e incertidumbre que se desprenden de esta infección; por ello se considera importante evaluar y describir el soporte social; no solo con fines teóricos, sino, además, para luego tener una herramienta con la cual diseñar programas de intervención en los diferentes niveles de la prevención.

El objetivo general de la presente investigación es identificar y describir el soporte social en un grupo de adultos seropositivos asintomáticos y sintomáticos. Investigaciones señalan que las personas con mayores índices de soporte social presentan menores probabilidades de experimentar emociones disfóricas, lo que se asocia con un mejor estatus de salud (Hays, Turner y Coates, 1992). Los objetivos específicos son identificar y describir los niveles de soporte social, así como establecer las posibles asociaciones entre las variables demográficas y los niveles de soporte social.

\section{Metodología}

\section{Nivel y tipo de investigación}

La presente investigación (Fasce, 1999) fue un estudio descriptivo que buscó identificar los niveles de soporte social en un grupo de adultos (Hernández, Fernández y Baptista, 1996). 


\section{Participantes}

El universo de estudio de la investigación estuvo conformado por la población de hombres y mujeres mayores de 18 años diagnosticados con VIH en Lima y que acuden a centros de salud, ONG o Grupos de Ayuda Mutua (GAM). Se excluyeron a aquellos portadores que presentaban trastornos psíquicos graves, es decir, que se hallaban especificados en el eje I del DSM IV y aquellos que se ubicaban en fase SIDA. El tipo de muestreo fue no probabilístico de tipo accidental.

La muestra estuvo constituida por 55 personas adultas, hombres y mujeres, de 18 a 58 años de edad, VIH positivas, asintomáticas y sintomáticas que se atendían en instituciones estatales de Lima. El $69 \%$ eran solteros, seguidos de los casados, el $14 \%$. Siendo la media de la edad 32 años para la muestra en general (Cuadro 1).

\section{Cuadro 1}

Estado civil y media de la edad de los participantes

\begin{tabular}{|lccc|}
\hline Estado civil & $f$ & $\%$ & $M$ \\
\hline Soltero & 38 & 69 & 32 \\
Casado & 8 & 14 & 32 \\
Viudo & 1 & 2 & 44 \\
Conviviente & 7 & 13 & 30 \\
Separado & 1 & 2 & 46 \\
\hline
\end{tabular}

Nota. $N=55$

Sobre el siguiente cuadro tenemos que el $60 \%$ de la muestra en general se halla entre los 24 y 35 años, así como el $66 \%$ de los hombres mientras que las mujeres tienden a encontrarse en los extremos, el $57 \%$ en el grupo de menores de 29 años, cumplen con esta característica solo el $37 \%$ de los hombres; asimismo, el $36 \%$ de las mujeres son mayores de 41 años, cumplien con esta característica el 9\% de los hombres (Cuadro 2). 
Soporte social en personas que viven con VIH

\section{Cuadro 2}

Rango de edades y sexo en porcentajes de los pe ticipantes

\begin{tabular}{|cccc|}
\hline Edad & $\begin{array}{c}\text { hombres } \\
\%\end{array}$ & $\begin{array}{c}\text { mujeres } \\
\%\end{array}$ & $\begin{array}{c}\text { Total } \\
\%\end{array}$ \\
\hline 23 o menos & 10 & 21 & 13 \\
$24-29$ & 27 & 36 & 29 \\
$30-35$ & 39 & 7 & 31 \\
$36-40$ & 15 & 0 & 11 \\
$41-46$ & 7 & 22 & 11 \\
$47-52$ & 2 & 7 & 4 \\
$53-58$ & 0 & 7 & 1 \\
\hline
\end{tabular}

Nota. $N=55$, mujeres $n=14$, hombres $n=41$

De los datos de la de la descripción de la muestra, los más relevantes son aquellos que señalan las características de las personas que participan en un GAM (Cuadro 3).

\section{Cuadro 3}

Participación en un GAM por orientación sexual en porcentajes

\begin{tabular}{|ccc|}
\hline Orientación Sexual & \multicolumn{2}{c|}{ Participa en GAM } \\
\hline & $\mathrm{Si}$ & No \\
Heterosexual & 48 & 52 \\
- mujeres & 50 & 50 \\
- hombres & 46 & 54 \\
Homosexual & 38 & 62 \\
- mujeres & 0 & 0 \\
- hombres & 38 & 62 \\
Bisexual & 29 & 71 \\
- mujeres & 0 & 0 \\
-hombres & 29 & 71 \\
\hline
\end{tabular}

Nota. $N=55$

De los hombres que tienen sexo con otros hombre (HSH) tenemos que la mayoría no participa en un GAM. Cabe resaltar que el $49 \%$ 
de la muestra es heterosexual, lo que refleja el veloz incremento de la seroprevalencia en esta población en los últimos años.

Luego tenemos que aquellos con menor grado de instrucción presentan menor probabilidad de participar (Figura 1).

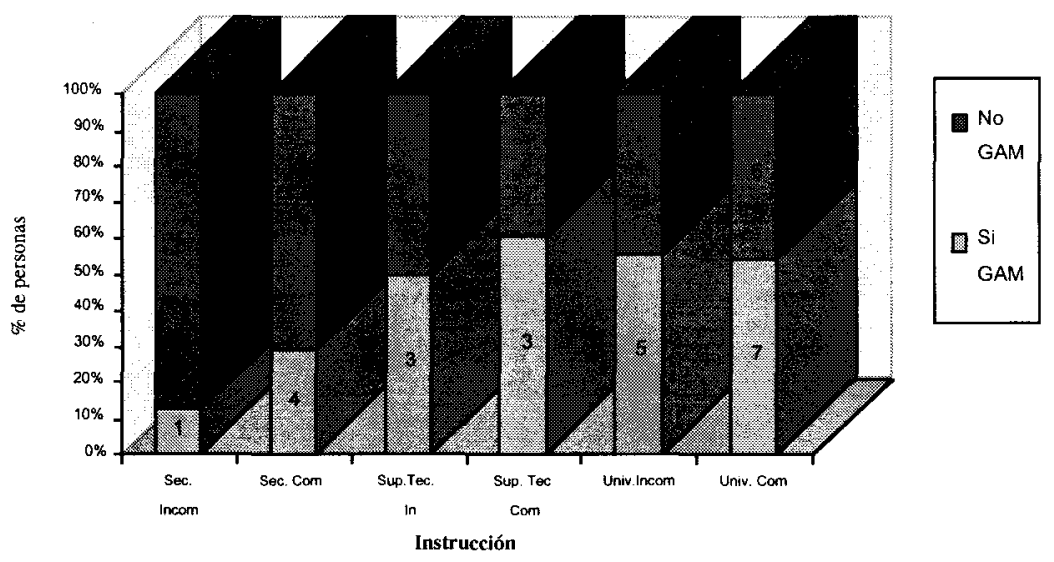

Figura 1. Participación en un GAM según nivel de instrucción.

\section{Instrumentos}

Los instrumentos usados fueron el Inventario de Encuestas de Soporte Social de Arizona (IESSA) y la Encuesta Demográfica. Ambos instrumentos fueron sometidos a criterio de jueces para obtener la validación de contenido. El IESSA ya fue validado por Rojas (1991), se obtuvo un Alpha de Cronbach de .52 para el soporte social percibido y de .72 para el efectivo, ambos fueron instrumentos, luego utilizados por Becerra (1993) y por Chavez-Ferrer (1995).

\section{Resultados}

En relación con los resultados en el Inventario de Entrevistas de Soporte Social de Arizona, se pudo observar que la percepción del 
tamaño de la red o soporte social percibido fue mayor $(M=6$ personas) a la red efectiva o soporte social efectivo ( $M=5$ personas); asimismo, el grado de necesidad de soporte social $(M=4)$ - mucha necesidad - fue ligeramente mayor a la satisfacción con este $(M=3)$ —indiferente-. Véase Cuadro 4.

\section{Cuadro 4}

Medias de los índices de soporte social

\begin{tabular}{|lll|}
\hline Índice del Soporte Social & $M$ & $D E$ \\
\hline Soporte social percibido & 6 & 2 \\
Soporte social efectivo & 5 & 3 \\
Satisfacción con el soporte & 3 & 6 \\
Necesidad de soporte & 4 & 4 \\
\hline
\end{tabular}

Nota. $N=55$.

Las medias de los índices del soporte social variaron según sexo y orientación sexual. Los índices del soporte social tuvieron en general la misma tendencia en mujeres y hombres heterosexuales; hubo mínimas diferencias en los índices. Los índices de soporte social en hombres homosexuales son comparativamente menores que la muestra con orientación heterosexual. En el caso de las mujeres, la media del tamaño de la red percibida fue de ocho personas $(M=8)$, al igual que en los hombres heterosexuales $(M=8)$ mientras que para los hombres homosexuales la media fue de cuatro personas $(M=4)$.

Asimismo, las medias del soporte social efectivo fueron de siete personas $(M=7)$, tanto para mujeres y hombres heterosexuales, y de tres personas $(M=3)$ para los hombres homosexuales.

Las mujeres y hombres heterosexuales presentaron un grado de necesidad de cuatro $(M=4)$-mucha necesidad-de soporte social mientras que los hombres homosexuales presentaron un grado de necesidad de tres $(M=3)$ - necesidad moderada- 
En cuanto a la satisfacción con el soporte, se observa que las mujeres y los hombres que tienen sexo con otros hombres (HSH) presentaron un grado de satisfacción de tres $(M=3)$-indiferencia- respecto de su soporte mientras que los hombres heterosexuales, de cuatro $(M=4)$-moderadamente satisfechos-. Véase Figura 2.

Los cuatro índices del soporte social fueron mayores para aquellos que sí participaron en un GAM. El soporte social percibido para aquellos que sí participan en un grupo de autoayuda fue en promedio de siete personas $(M=7)$ mientras que para los que no participaron fue de seis $(M=6)$. El soporte social efectivo o tamaño de la red que efectivamente brindó soporte fue de cinco $(M=5)$ para ambas poblaciones.

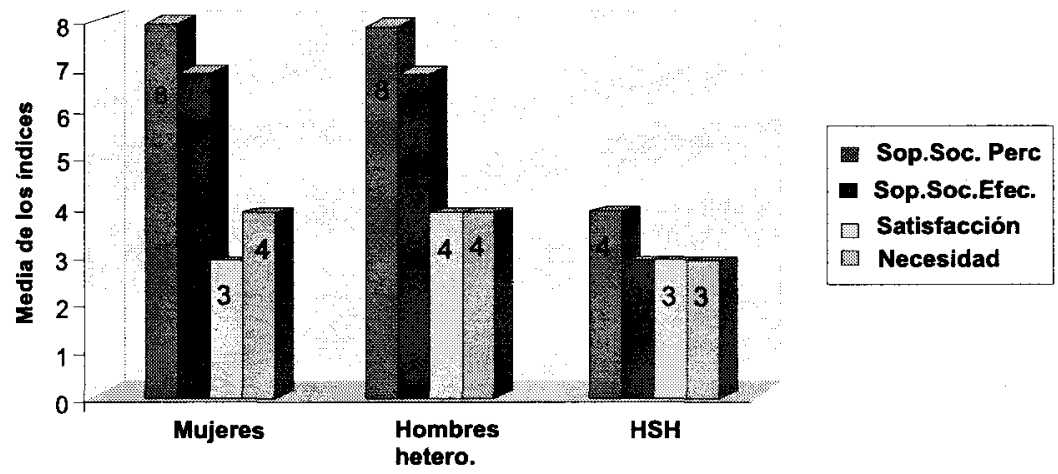

Figura 2. Índices del IESSA por orientación sexual y sexo.

La necesidad de soporte social para aquellos que sí participan en un GAM fue de cuatro $(M=4)$ - mucha necesidad - mientras que para aquellos que no lo hacen, la necesidad fue de tres $(M=3)$ - necesidad moderada-. En ambos grupos, la satisfacción fue de tres (indiferente). Véase Figura 3.

Las medias de los índices de soporte social para la función interacción íntima mantuvieron similar comportamiento que los índices 
Soporte social en personas que viven con VIH

generales, el tamaño de la red percibida $(M=2)$ fue ligeramente mayor que la efectiva $(M=2)$ y el grado de satisfacción $(M=4)$ similar a la necesidad $(M=4)$. Cuadro 5 .

\section{Cuadro 5}

Distribución de los índices del soporte social en la función interacción íntima

\begin{tabular}{|lcc|}
\hline $\begin{array}{l}\text { Índices del soporte social en la } \\
\text { función interacción íntima }\end{array}$ & $M$ & $D E$ \\
\hline Soporte social percibido & 2 & 1 \\
Soporte social efectivo & 2 & 1 \\
Satisfacción con el soporte & 4 & 1 \\
Necesidad de soporte & 4 & 1 \\
\hline
\end{tabular}

Nota. $N=55$.

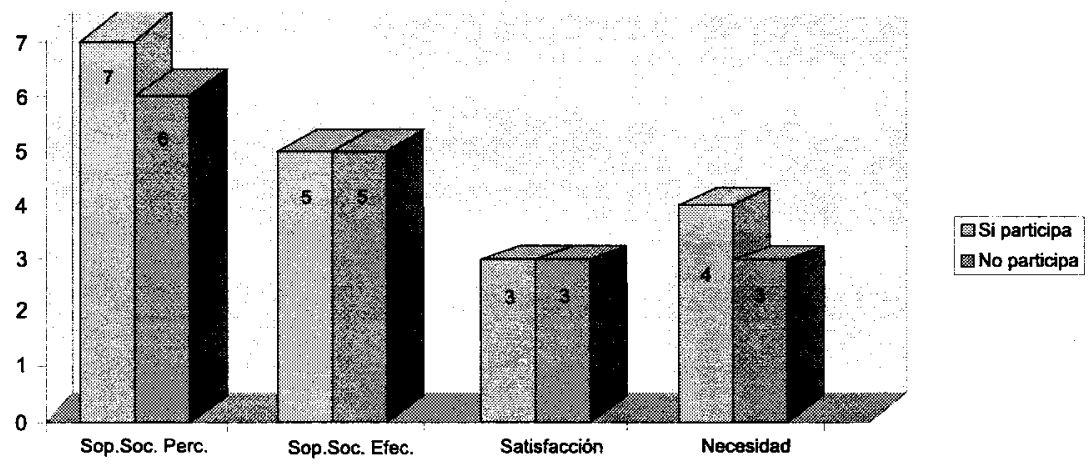

Figura 3. Índices del IESSA según participación en GAM.

En el caso de la función de ayuda material, se observa que la red percibida fue mayor $(M=2)$ que la efectiva $(M=1)$. La satisfacción ( $M=3$ ) fue menor que la necesidad $(M=4)$ lo que significa que los participantes se hallaron insatisfechos (Cuadro 6). 


\section{Cuadro 6}

Distribución de los índices de soporte social en la función ayuda material

\begin{tabular}{|lcc|}
\hline $\begin{array}{l}\text { Índices del soporte social en } \\
\text { la función ayuda material }\end{array}$ & $M$ & $D E$ \\
\hline Soporte social percibido & 2 & 1 \\
Soporte social efectivo & 1 & 1 \\
Satisfacción con el soporte & 3 & 3 \\
Necesidad de soporte & 4 & 1 \\
\hline
\end{tabular}

Nota. $N=55$.

La función de consejo e información del soporte social presentó un comportamiento bastante uniforme respecto del índice anterior (Cuadro 7), donde su percepción $(M=2)$ fue similar al real $(M=1)$ y la satisfacción $(M=3)$ fue menor que la necesidad $(M=4)$.

\section{Cuadro 7}

Distribución de los índices del soporte social en la función consejo e información

\begin{tabular}{|lcc|}
\hline $\begin{array}{l}\text { Índices del soporte social en la } \\
\text { función consejo e información }\end{array}$ & $M$ & $D E$ \\
\hline Soporte social percibido & 2 & 1 \\
Soporte social efectivo & 1 & 1 \\
Satisfacción con el soporte & 3 & 1 \\
Necesidad de soporte & 4 & 1 \\
\hline
\end{tabular}

Nota. $N=55$.

En lo que a la información positiva se refiere, el tamaño de la red percibida $(M=3)$ fue, en promedio, superior a la efectiva $(M=1)$. La satisfacción $(M=3)$ y la necesidad $(M=3)$ estuvieron en similares condiciones (Cuadro 8). 
Soporte social en personas que viven con VIH

\section{Cuadro 8}

Distribución de los índices del soporte social en la función información positiva

\begin{tabular}{|lcc|}
\hline Índices del soporte social en la & $M$ & $D E$ \\
\hline función información positiva & 3 & 3 \\
Soporte social percibido & 1 & 1 \\
Soporte social efectivo & 3 & 2 \\
Satisfacción con el soporte & 3 & 1 \\
Necesidad de soporte & & \\
\hline
\end{tabular}

Nota. $N=55$.

El promedio de la muestra percibió una red social $(M=1)$ similar a la que efectivamente $(M=1)$ tiene en lo que a la función de asistencia del soporte se refiere, se encontró una equivalente satisfacción $(M=4)$ y necesidad $(M=4)$ (Cuadro 9).

El promedio de la muestra percibió una red $(M=3)$ de participación social que efectivamente presentó $(M=3)$ y se encontraron en ella a moderadamente satisfechos con ella $(M=4)$ y con mucha necesidad $(M=4)$ (Cuadro 10).

\section{Cuadro 9}

Distribución de los índices del soporte social en la función asistencial

\begin{tabular}{|lcc|}
\hline Índices del soporte social en & $M$ & $D E$ \\
\hline la función asistencial & 1 & 1 \\
\hline Soporte social percibido & 1 & 1 \\
Soporte social efectivo & 4 & 1 \\
Satisfacción con el soporte & 4 & 1 \\
Necesidad de soporte & \\
\hline
\end{tabular}

Nota. $N=55$. 


\section{Cuadro 10}

Distribución de los índices del soporte social en la función de participación social

\begin{tabular}{|lcc|}
\hline Índices del soporte social en & & \\
la función de participación social & $M$ & 2 \\
\hline Soporte social percibido & 3 & 2 \\
Soporte social efectivo & 3 & 1 \\
Satisfacción con el soporte & 4 & 1 \\
Necesidad de soporte & 4 & 2 \\
\hline
\end{tabular}

Nota. $N=55$.

Los índices de soporte social fueron correlacionados con las variables demográficas de participación en un Grupo de Ayuda Mutua, sexo, orientación sexual y sexo, tiempo de diagnóstico, estatus de la enfermedad y edad.

La orientación sexual y el sexo presentan una gran asociación con el soporte social percibido y efectivo $(r=.60$ y $r=.65$, respectivamente), es decir, el tamaño de la red, tanto la percibida como la efectiva varían significativamente, dependiendo de si se es mujer, hombre homosexual u hombre heterosexual. Ocurre lo mismo con la satisfacción con el soporte $(r=.45)$ y la necesidad de él $(r=.37)$.

La variable sexo tuvo una asociación media con el tamaño de la red percibida $(r=-.39)$, el tamaño de la red efectiva $(r=-.39)$, así como con la necesidad de soporte social $(r=-.32)$, es decir, estos índices del soporte varían según el sexo al que pertenezca la persona.

La participación en un grupo de autoayuda tuvo una asociación pequeña $(r=.25)$ con el grado de necesidad de soporte social, es decir, el pertenecer a un grupo de autoayuda incrementaría la demanda de soporte en la muestra.

El tiempo de conocimiento del diagnóstico de seropositividad tuvo una correlación media y positiva con la necesidad de soporte social ( $r$ 
Soporte social en personas que viven con VIH

$=.30$ ), es decir, a mayor tiempo de diagnosticado más necesidad de soporte social.

El presentar o no alguna enfermedad asociada al diagnóstico y la edad no correlacionaron con ninguno de los índices del soporte social (Cuadro 11).

\section{Cuadro 11}

Asociaciones del IESSA con las variables demográficas

\begin{tabular}{|lcccccc|}
\hline \multicolumn{1}{|c}{ IESSA } & $\begin{array}{c}\text { Participación } \\
\text { en un } \\
\text { GAM }\end{array}$ & Sexo & $\begin{array}{c}\text { Orientación } \\
\text { sexual y } \\
\text { sexo }\end{array}$ & $\begin{array}{c}\text { Tiempo } \\
\text { de } \\
\text { diagnóstico }\end{array}$ & Sintomatología Edad \\
\hline $\begin{array}{l}\text { Soporte social } \\
\text { percibido }\end{array}$ & .22 & $-.39 *$ & $.60 *$ & .13 & .04 & .09 \\
$\begin{array}{l}\text { Soporte social } \\
\text { efectivo }\end{array}$ & .11 & $-.39 *$ & $.65 *$ & .04 & -.12 & .04 \\
$\begin{array}{l}\text { Satisfacción con } \\
\text { Soporte }\end{array}$ & .12 & -.06 & $.45 *$ & -.12 & -.01 & -.11 \\
$\begin{array}{l}\text { Necesidad de } \\
\text { soporte }\end{array}$ & $.25 *$ & $-.32 *$ & $.37 *$ & $.30 *$ & -.11 & .07 \\
\hline
\end{tabular}

Nota. $N=55 . * p<.05$

\section{Discusión}

Se ensayó una aproximación a la comprensión del soporte social en personas seropositivas. El interés vino determinado por la conveniencia de apoyar a estas personas debido a que la literatura señala que un adecuado soporte social repercute en menores niveles de distrés. Se ha señalado que la presencia de niveles elevados de ansiedad y depresión ejercen efectos negativos sobre la salud y la respuesta ante los tratamientos (Antoni et al., 1991; Rabkin et al., 1991 y Kemmeny et al., 1994). De aquí la importancia de conocer cómo se influencian las diferentes variables que afectan la salud, principalmente en los seropositivos pues es la población con salud más frágil. 
Ello permitirá una mejor definición de los alcances y de la metodología del apoyo psicológico.

La muestra está constituida por adultos de ambos sexos entre 18 y 58 años. Asimismo, el rango de edades se aproxima al $75 \%$ de personas VIH positivas entre 20 y 39 años propuesto para el Perú por la Organización Panamericana de la Salud (1999); cabe destacar que en este rango de edad, la mayoría de personas se encuentran trabajando. Ulin (1992) señala la importancia de considerar las consecuencias económicas de la epidemia a nivel social, pues no solo el seropositivo pierde capacidad de trabajar, sino que genera gastos a su entorno cercano y a la sociedad.

Referencialmente, la proporción hombre-mujer de la muestra obtenida se aproxima a la estadística oficial (3:1) - Ministerio de Salud, 1999-. Las mujeres constituyen el $26 \%$ de la muestra, lo que confirma las observaciones respecto de la progresiva feminización de la epidemia por lo que se las considera grupo vulnerable (Kornblit, 1997, CONTRASIDA, 1998a). Para aquellas que tienen prole, la maternidad y cuidado futuro de los hijos generan preocupación respecto del futuro.

En este sentido, se ha visto que las mujeres tienen fortaleza para afrontar sus dificultades mientras tienen hijos menores de cuatro años (Torres, 1998) debido a que la motivación de nutrir y cuidar a los demás es mayor que cuando los hijos asisten a la escolaridad formal - cuando son más independientes - por lo que se da una tendencia a que la motivación sea de logro (Ragúz, 1990), aspectos que son difíciles de satisfacer si se es VIH positiva.

A partir de la literatura consultada y por la experiencia de trabajo con mujeres seropositivas se sabe que la mayoría de estas es contagiada por su pareja estable, en su hogar (Torres, 1998). En este estudio ninguna de las mujeres de la muestra ejercía como trabajadora sexual, eso coincide con las investigaciones que las ubican como grupo de reducido 
índice de riesgo de contagio. Eso contradice la creencia general que las ubica como muy expuestas (Estebañez et al., 1992).

Respecto de la orientación sexual, la muestra refleja el veloz incremento de seroprevalencia entre la población heterosexual en los últimos años (Gómez, 1997). Los contagios por vía heterosexual en el Perú entre 1995 y 1997 son del 53\% mientras que los contagios por vía homosexual son el $43 \%$. En la muestra los casos de heterosexuales alcanzan el $49 \%$ mientras que los homosexuales son el $51 \%$.

En relación con el soporte social, encontramos que los resultados del IESSA varían según sexo, orientación sexual y asistencia o no a un GAM. Los heterosexuales, hombres y mujeres, presentan índices de soporte social mayores que la media general, se resalta el índice de satisfacción en los hombres heterosexuales, que es más elevada, mientras que los HSH presentan tres de los cuatro índices del soporte social por debajo del promedio, la satisfacción en ellos es igual a la media general. Esto coincide con los trabajos de Kelly y St. Lawrence (1986) donde señalan el rechazo del que son objeto los HSH como un factor importante en la disminución del soporte social.

Respecto de la participación en un grupo de ayuda mutua, son aquellos que participan los que tienen mayores redes de soporte social tanto percibido como efectivo y se hallan más satisfechos. Es interesante señalar que también son los que presentan más necesidad.

En las funciones del soporte social, la media de la población presenta similares índices de satisfacción y necesidad, con excepción de las funciones de ayuda material y consejo e información donde la insatisfacción es la media de la muestra. Este dato cabría tomarlo en cuenta pues las personas que viven con VIH demandan de información para evitar la incertidumbre frente a la enfermedad; la carencia de ella es la que impide que se tenga conductas preventivas hacia la reinfección y el cuidado de los otros. El que la función de ayuda 
material no se halle satisfecha se entiende si tomamos en cuenta que la muestra proviene de un sector socioeconómico medio bajo y bajo y el tratamiento para el VIH es costoso. Hays et al. (1992) señalan que estas dos funciones consejo e información y ayuda material junto a la función de interacción íntima están inversamente correlacionados con la depresión.

Sobre las asociaciones entre las variables demográficas y el IES$S A$, encontramos que la orientación sexual y el sexo se asocian en la muestra fuertemente con las variaciones en los índices del soporte social, son las mujeres y hombres heterosexuales los que presentan mayores redes de soporte social percibido y efectivo, los hombres heterosexuales la mayor satisfacción, compartiendo los grados de necesidad con las mujeres; ellas comparten el grado de satisfacción con los hombres que tienen sexo con otros hombres (HSH).

En el caso de los HSH se observa que son los que presentan las redes de soporte más pequeñas, menor satisfacción y menor necesidad. El que presenten menores redes de soporte se puede entender por la doble estigmatización que sufren por ser VIH positivo y tener una orientación sexual no convencional (López, 1996). Debido a esta estigmatización, los HSH suelen formar redes donde todos son homosexuales (acuden a bares, cafés y discotecas que denominan de ambiente) y muchos de ellos viven con VIH, por lo que es usual que vean morir a sus amigos, lo cual los deprime y resta fuentes de soporte (Turner, 1983).

Kelly y St. Lawrence (1986) sostienen que la representación social negativa hacia los HSH se debe al desconocimiento del estilo de vida gay. Las representaciones sociales vigentes, desde los inicios de la epidemia, persisten en considerar a la población homosexual, epidemiológicamente $\mathrm{HSH}$, como grupos vulnerables, esto a pesar de que la evolución de la epidemia pareciera demostrar lo contrario, ya que lo que parece ocurrir es que toda persona con una vida sexual activa es vulnerable al VIH. El que se siga asociado a los HSH con la expansión de la epidemia puede deberse al rol que juegan en la 
memoria las representaciones sociales; las personas con una representación social negativa frente a la homosexualidad negarán la información que contradiga sus esquemas para evitar la disonancia cognitiva (Morales et al., 1997).

Durante la XII Conferencia Internacional sobre SIDA, realizada en Ginebra, se recomendó que para el logro del objetivo de información y educación sobre sexo seguro en HSH se debía brindar información sobre las medidas de prevención, pero también se debían abarcar acciones para elevar la autoestima, internalizar la homofobia, desarrollar habilidades de comunicación y de manejo de las relaciones interpersonales pues todos ellos son factores que intervienen en la adopción de conductas de cuidado (CONTRASIDA, 1998b); el que se recalque que falta trabajar habilidades de comunicación y manejo de las relaciones interpersonales nos indica que es una población que las presenta en déficit, lo que aunado a la estigmatización les dificultaría tener un buen soporte social.

El tener más tiempo de diagnosticado se relaciona con la cercanía a la muerte; al respecto, Kelly, Murphy, Sikkerman y Kalichman (1993) señalan que el acercarse a la muerte incrementa la necesidad de relaciones interpersonales significativas y suaviza las barreras pasadas del soporte familiar

El participar en un GAM presenta una significativa asociación en la muestra con el incremento de la necesidad de soporte social; al respecto se ha visto que es importante analizar el papel de enfermo con el que se identifica la persona que vive con VIH. Ellos exigen cuidado, en algunos casos se sienten liberados de responsabilidades sociales, como el trabajo. Estas reuniones estimulan una mayor dependencia con la enfermedad, los ayuda a vivir con ella y proporcionan consejos prácticos para la vida, así como la dependencia económica al facilitar la obtención de ayuda y beneficios a los miembros del grupo (Chavez-Ferrer, 1995). 
Las personas que viven con el VIH se identifican con su papel de enfermo y adoptan una actitud de espera de soluciones y no de acción. Dentro del grupo se habla de sus derechos, de lo que la sociedad le debe dar, lo que acrecienta su necesidad de soporte social, sus expectativas, una variable exógena a este comportamiento es la filosofía de las asociaciones que luchan constantemente por el mejoramiento de la calidad de vida de los mismos. Esto influye en la actitud de los portadores al creer que tienen más derechos en la atención de salud que otros enfermos.

Es recomendable analizar el rol de los GAM como dadores de soporte, desde su efectividad aplicando, tal vez, el IESSA y el IDB al ingreso del portador y contrastándolo después de un período de tiempo pertinente de participación. Un aporte a estos grupos sería un estudio de las motivaciones que los impulsan a unirse a los grupos y de los factores que determinan los niveles de participación de sus miembros, ya sea permanente, eventual o ausente.

En los trabajos realizados por Kelly, St. Lawrence, Hood y Brasfield (1989) se llegó a la conclusión que las intervenciones clínicas que empleaban grupos de soporte, comprometiéndolos en grupos de soporte comunitarios disponibles, ayudaban a los individuos a establecer o restablecer relaciones con amigos y familiares, mejoraba sus estrategias de afrontamiento, y, por lo tanto, disminuían sus niveles de depresión. Por ello, es recomendable fomentar la participación en estos grupos tomando en cuenta lo antes discutido.

Según diversas investigaciones, se ha podido concluir que el presentar un adecuado soporte social disminuye los efectos negativos producto de estresores psicosociales, pues este nos permite reducir el estado emocional negativo, obtener asistencia financiera, información, consejo, participación social y ayuda en los quehaceres diarios (Alvarado, 1985; Hays et al., 1992; Sarason, Sarason y Pierce,1990; Kelly, 1987; Kelly y St. Lawrence, 1986; Kelly, St. Lawrence, Hood y Brasfield, 1989; Kelly et al., 1993; Newcomb, 1990). 
Sería recomendable ampliar los estudios sobre la variable estudiada en la población femenina debido a que se comporta de manera diferente y el número de seropositivas al VIH se incrementa permanentemente, lo cual trae como consecuencia que existan casos de transmisión vertical (madre-niño) siendo el porcentaje $0.6 \%$ del total de gestantes en Lima. La mejor manera de prevenir la transmisión vertical sería reducir la infección en las mujeres en edad fértil, lo cual demanda de campañas de prevención focalizadas en ellas (CONTRASIDA, 1998a; Castro, 1998).

Se considera necesario ampliar las investigaciones, principalmente, con la población heterosexual pues la mayor parte de las investigaciones sobre el tema se realizan con una muestra de HSH y, por lo que hemos podido observar, las necesidades no son iguales para los diferentes grupos de la muestra; las mujeres requieren de un trabajo diferente al de los hombres y las necesidades de ellos difieren según su orientación sexual. Es por ello que se propone hacer programas de prevención por separado y que las intervenciones sean diferentes para cada grupo. Asimismo, sería idóneo se realicen investigaciones longitudinales e interdisciplinarias, donde se evalúe cómo el estatus de salud varía según varían otras áreas como el soporte social, el ánimo, los estilos de afrontamiento, el manejo de la cólera, así como su relación con la personalidad. El conocer cómo interactúan las diferentes características de la población nos daría una visión más amplia de ella y las intervenciones podrían ser más cercanas a sus necesidades.

\section{Referencias}

American Psychiatric Association (1994). Diagnostic and statistical manual of mental disorders (4ta. ed.). Washington DC: autor. Alvarado, O. (1985). Soporte social: ¿teoría o práctica? Cuadernos de Psicología, 4, 1-2. 
Antoni, M. H., Bagett, L., Ironson, G., La Perriere, A., August, S., Klimas, N., Schneiderman, N. y Fletcher, M. A. (1991). Cognitive-behavioral stress management interventions buffers distress responses and immunologic changes fallowing notification of HIV-1 seropositivity. Journal of Consulting and Clinical Psychology, 59(6), 906-915.

Becerra, S. (1993). Factores Demográficos y psicosociales asociados a la decisión de continuar o terminar con el embarazo en un grupo de adolescentes de estrato socioeconómico bajo. Tesis para optar el título de licenciatura en Psicología Clínica, Pontificia Universidad Católica del Perú, Lima.

Castro, O. P. (1998, mayo 10). Informe: Aumenta la esperanza de vida para hijos de portadoras del VIH. El Comercio, p.18.

CONTRASIDA (1998a). Contrasida Informa. Boletín informativo del Programa de Enfermedades de Transmisión Sexual y SIDA, 7, 9.

CONTRASIDA (1998b). Contrasida Informa. Boletín informativo del Programa de Enfermedades de Transmisión Sexual y SIDA, 7, 10.

Chavez Ferrer, L. (1995). Soporte Social en pacientes VIH positivos. Memoria para optar el grado de Bachiller en Humanidades con mención en Psicología, Pontificia Universidad Católica del Perú, Lima.

Estebañez, P., Rua-Figueroa, M., Aguilar, M., Fitch, K., Palacios, V., Pelez, L. y Nájera, R. (1992). Prevalencia de VIH y factores de riesgo entre prostitutas hispanas. Ponencia presentada en la VII Conferencia Internacional sobre SIDA, Amsterdam.

Fasce, N. (1999). Soporte social y depresión en personas adultas que viven con VIH. Tesis de licenciatura para optar el grado de licenciatura en Psicología, Pontificia Universidad Católica del Perú, Lima.

Gómez, H. D. (1997, Junio). SIDA y ETS: Qué se ha hecho y qué se debe hacer. Ponencia presentado en el IV Congreso Latinoamericano de Ciencias Sociales y Medicina, Coco y Mor, México. 
Hays, R. B., Turner, H. y Coates, T. (1992). Social Support, AIDSRelated symptoms, and Depression among gay men. Journal of Consulting and Clinical Psychology,3, 463-469.

Hernández, R., Fernández, C. y Baptista, P. (1996). Metodología de la Investigación. México: McGraw-Hill.

Kelly, J . (1987). Stigmatization of AIDS patients by physicians. American Journal of Public Health. 77 (7), 789-791.

Kelly, J., Murphy, D., Sikkerman, K., Kalichman, J. C. (1993).

Psychological interventions to prevent HIV infection are urgently needed. American Psychologist, 48 (10), 1023-1034.

Kelly, J., St.Lawrence, J. S. (1986). Behavioral Intervention and AIDS. The Behavioral Therapist, 9 (6), 121-125.

Kelly, J., St. Lawrence, J. S., Hood, H. y Brasfield, B. (1989).

Behavioral intervention to reduce AIDS risk activities. Journal of consulting and clinical psychology. 57 (1), 60-67.

Kemmeny, M. E., Weiner, H., Taylor, S. E., Schneider, S., Visscher, B. y Fahey, J. L. (1994). Repeated bereavement, depressed mood, and immune parameters in HIV seropositive and seronegative gay men. Health Psychology, 13, 14-24.

Kornblit, A. L. (1997, Junio). SIDA y ETS:Qué se ha hecho y qué se debe hacer. Ponencia presentada en el IV Congreso Latinoamericano de Ciencias Sociales y Medicina, Coco y Mor, México.

López, L. (1996, setiembre 5). Salud reproductiva y prevención de ETS y SIDA. Taller de Educación Sexual para Comunicadores Sociales (Ministerio de Educación), Lima.

Morales, J. F., Moya, M., Rebolloso, E., Fernández, J. M., Huici, C., Marques, J., Páez, D. y Pérez, J. A. (1997). Psicología social. Madrid: McGraw-Hill.

Ministerio de Salud (1999). Información estadística 1983 - Enero 1999 del programa de enfermedades de transmisión sexual y SIDA (PROCETSS). Lima: autor.

Newcomb, M. (1990). What structural equation modeling can tell us about social support. En B. Sarason, I. Sarason y G. Pierce 
(Eds.), Social support: An international view (pp. 26-60). Washington: Wiley.

Organización Mundial de la Salud (1998, noviembre). Weekly Epidemiological Record 48, 27.

Organización Panamericana de la Salud (1999, marzo). Vigilancia del SIDA en las Américas. Informe trimestral del Programa Regional del SIDA/ETS-División de prevención y control de enfermedades, no publicado.

Rabkin, J. G., Williams, J. B. W., Remien, R. H., Goetz, R. R., Kretzner, R. y Gorman, J. M. (1991). Depression, lymphocyte subsets, and human immunodeficiency virus symptoms on two occasions in HIV-positive homosexual men. Archives of General Psychiatry, 48, 111-119.

Ragúz, M. (1990). Maternidad/maternalidad y trabajo: "Efectos" del rol dual sobre las madres que trabajan. Revista de Psicología de la PUCP, 8 (2), 181-197.

Rojas, R. (1991). Perfil psicosocial de la adolescente gestante de estrato socioeconómico bajo. Memoria para optar el grado de Bachiller en Humanidades con mención en Psicología, Pontificia Universidad Católica del Perú, Lima.

Torres, G. (1998). Oye nuestras voces. ICW Noticias, 7, 2.

Turner, R. J. (1983). Direct, indirect unmotivating effect of social support on psychological distress and associated conditions. En H. B. Kaplan (Ed.), Psychosocial stress: trends in theory and research (pp.105-155). Nueva York: Academic Press.

Ulin, U. (1992). African human and AIDS: negociating behavior change. Social Science and Medicine, 34, 63-73. 